\title{
Sparse Representation of FHSS Signals in the Hermite Transform Domain
}

\author{
Miloš Brajović, Student Member, IEEE, Andjela Draganić, Student Member, IEEE, Irena Orović, \\ Member, IEEE, and Srdjan Stanković, Senior Member, IEEE
}

\begin{abstract}
Signal sparsity is exploited in various signal processing approaches. Signal compression, classification, coding, as well as the recently introduced compressed sensing are some examples where the possibility to represent a signal sparsely determines the efficiency of the applied processing technique. However, the possibility of a sparse signal representation in a transform basis is highly dependent on the signal nature. Therefore, finding a suitable basis where the signal exhibits a compact support is a challenging task. In this paper, the Hermite Transform (HT) is considered as a sparsity domain for the FHSS wireless communication signals. The transform coefficients sparsification is done by optimizing the scaling factor and time-shift of basis functions. The optimization is done by minimizing the concentration measure of HT coefficients. The theory is verified by numerical examples with synthetic FHSS signals.
\end{abstract}

Keywords - FHSS signals, signal sparsity, sparsification, Hermite transform domain.

\section{INTRODUCTION}

$\mathrm{S}$ PARSE signal representation in a certain domain is commonly desirable in both signal processing and analysis [1]-[7]. A signal can be considered sparse in a certain transform domain if it can be represented with a small number of non-zero coefficients [1], [6]. Additionally, signals having a small number of significant coefficients while the influence of other coefficients is negligible although they are not zeros, can be considered as approximately sparse. This means that the signal energy is concentrated within a small number of coefficients in the sparsity domain [5], [6]. This important property has been already exploited in various compression algorithms, for example, MPEG and JPEG, in order to remove redundancy and compress the signal [5].

During the last decade, a new signal processing area, known as Compressive Sensing (CS) [2]-[7], has been

Paper received June 5, 2017; revised October 13, 2017; accepted October 14, 2017. Date of publication December 25, 2017. The associate editor coordinating the review of this manuscript and approving it for publication was Prof. Branimir Reljin.

This paper is a revised and expanded version of the paper presented at the 24th Telecommunications Forum TELFOR 2016 [15].

This work is supported by the Montenegrin Ministry of Science, project grant funded by the World Bank loan: CS-ICT "New ICT Compressive sensing based trends applied to: multimedia, biomedicine and communications".

Authors are with the Faculty of Electrical Engineering, University of Montenegro, Cetinjski put bb, 81000 Podgorica, Montenegro Corresponding author is Andjela Draganić (phone: +382-63-218-048; e-mail: andjelad@ac.me). developed exploiting the signal sparsity in some transformation domain. Namely, such sparse signals can be reconstructed from a reduced number of signal samples (measurements) taken in the domain where the signal is dense. This number can be significantly smaller than the number of samples obtained following the sampling theorem, requiring the sampling frequency at least two times higher than the maximal signal frequency.

The algorithms for recovering the missing information in sparse signals are constantly developing. The missing samples may arise as a consequence of their physical unavailability, intentional sampling strategy, or they are omitted due to a high noise influence. In certain biomedical applications, the number of measurements is intentionally reduced in order to avoid the patients' exposure to radiation, etc.

Signals can be sparse in various transformation domains. Sparse representation can be achieved by choosing an appropriate transform basis, i.e. by decomposing the signal into the set of suitable expansion functions. Depending on the signal nature, sparsity domains include the discrete Fourier transform (DFT), discrete cosine transform (DCT), Hermite transform (HT) etc. [1], [2], [5].

The main topic of this paper is the problem of choosing the optimal parameters for the sparse representation of frequency hopping spread spectrum (FHSS) signals in the HT domain [8]-[20]. The HT is used in analysis of various signals - biomedical signals (EEG), ultra wideband (UWB) and communication signals, signals in computer tomography, etc. It has many desirable properties [12], [15]. Having in mind that majority of these signals can be modelled with a smaller number of Hermite functions compared to the signal length, the HT finds usage in compression algorithms [12], [13]. Signals have a potential to be sparsely represented in the HT domain if they exhibit a certain level of resemblance with the Hermite basis functions [13].

The FHSS modulated signals, consisted of short duration sinusoidal components [8], [10], [11], [21], are well known for the spectrum leakage around the frequencies of the signal components in the DFT domain. The HT is considered due to the fact that the Hermite functions show a similar time-domain shape as the FHSS signal components. Optimal fitting between the signal components and expansion functions can be achieved using optimization of the time-scaling factor and time-shift parameter of the Hermite basis functions [12]. The main goal of this optimization procedure is to concentrate the signal into the smallest possible number of HT coefficients. In [18], the Hermite transform domain was identified as a potential domain of sparsity for FHSS signals. The goal was 
to find a suitable sparsity domain, which is important for later application of the CS approach, or any other sparsitybased approaches. In this paper, initial results presented in [18] are expanded. The algorithm for the gradient-based concentration measure minimization applied in automatic scaling factor optimization [12] is revisited. In this paper, the comprehensive description of the algorithm is presented, with substantial details regarding the implementation and parameter initialization. Particular attention is given to the interpretation of the scaling factor influence on the non-uniform time-axis, indicating that the presented approach automatically places the non-uniform sampling points in the vicinity of corresponding points of the uniform grid obtained in accordance with the sampling theorem. This indicates that signal resampling from the uniform to the non-uniform grid required by the HT retains the original signal waveform in the best possible way.

The paper is organized as follows: Section II is the theoretical background on the HT. The procedure for the HT optimization is described in Section III, while the experimental results are given in Section IV. The paper ends with concluding remarks.

\section{THEORETICAL BACKGROUND}

The $M$-th order Hermite polynomials are defined as

$$
H_{M}(t)=(-1)^{M} e^{t^{2}}\left(d^{M}\left(e^{-t^{2}}\right) / d t^{M}\right) .
$$

They are closely related with the Hermite functions. Discrete Hermite function of order $p$ is defined as [9], [13][20], [22]:

$$
\psi_{p}(t)=\frac{e^{-\frac{t^{2}}{2 \sigma^{2}}} H_{p}(t / \sigma)}{\sqrt{\sigma 2^{p} p ! \sqrt{\pi}}},
$$

and also with the following recursion [5]:

$$
\begin{aligned}
& \psi_{0}(t)=\frac{1}{\sqrt[4]{\pi}} e^{-\frac{t^{2}}{2 \sigma^{2}}}, \quad \psi_{1}(t)=\frac{\sqrt{2} t}{\sqrt[4]{\pi}} e^{-\frac{t^{2}}{2 \sigma^{2}}}, \\
& \psi_{p}(t)=t \sqrt{\frac{2}{p}} \psi_{p-1}(t)-\sqrt{\frac{p-1}{p}} \psi_{p-2}(t), \forall p \geq 2 .
\end{aligned}
$$

Scaling factor $\sigma$ is used to match functions to the signal, by stretching or compressing them. In terms of continuoustime Hermite expansion, a continuous-time signal $f(t)$ can be represented as [5], [13], [18]:

$$
f(t)=\sum_{p=0}^{\infty} c_{p} \psi_{p}(t)
$$

where $\psi_{p}(t)$ is used to denote the Hermite functions, whereas $c_{p}$ denotes the Hermite expansion coefficients. The discrete Hermite transform requires the sampling of basis functions and the signal at points proportional to the roots of the $M$-th order Hermite polynomial (1). In that case, expansion (4) becomes finite, with exactly $M$-terms. For signals already sampled uniformly according to the sampling theorem, a finite sinc interpolation can be done in order to obtain signal values at the points of interest. The interpolation error for signals with a finite time-support is negligible in that case [12].

Hermite coefficients are calculated using the GaussHermite quadrature [5], [12]:

$$
c_{p} \approx \frac{1}{M} \sum_{m=1}^{M} \frac{\psi_{p}\left(t_{m}\right)}{\left[\psi_{M-1}\left(t_{m}\right)\right]^{2}} f\left(t_{m}\right),
$$

where the sampling at points $t_{m}$ proportional to the roots of the $M$-th order Hermite polynomial is assumed. In matrix form, the discrete HT is defined as follows:

$$
\left[\begin{array}{c}
c_{0} \\
c_{1} \\
\cdots \\
c_{P-1}
\end{array}\right]=\frac{1}{M}\left[\begin{array}{ccc}
\frac{\psi_{0}\left(t_{1}\right)}{\left(\psi_{M-1}\left(t_{1}\right)\right)^{2}} & \cdots & \frac{\psi_{0}\left(t_{M}\right)}{\left(\psi_{M-1}\left(t_{M}\right)\right)^{2}} \\
\frac{\psi_{1}\left(t_{1}\right)}{\left(\psi_{M-1}\left(t_{1}\right)\right)^{2}} & \cdots & \frac{\psi_{1}\left(t_{M}\right)}{\left(\psi_{M-1}\left(t_{M}\right)\right)^{2}} \\
\ldots & \cdots & \ldots \\
\frac{\psi_{M-1}\left(t_{1}\right)}{\left(\psi_{M-1}\left(t_{1}\right)\right)^{2}} & \cdots & \frac{\psi_{M-1}\left(t_{M}\right)}{\left(\psi_{M-1}\left(t_{M}\right)\right)^{2}}
\end{array}\right]\left[\begin{array}{c}
f\left(t_{1}\right) \\
f\left(t_{2}\right) \\
\ldots \\
f\left(t_{M}\right)
\end{array}\right],
$$

where $\mathbf{c}$ and $\mathbf{f}$ are Hermite coefficients and signal vectors, $\mathbf{H}$ is the transform matrix. The inverse transform reads:

$$
\begin{aligned}
& \mathbf{f}=\mathbf{H}^{-1} \mathbf{c},
\end{aligned}
$$

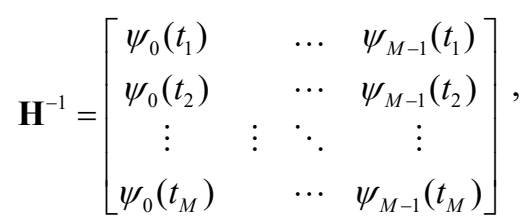

with $\mathbf{H}^{-1}$ being the inverse HT matrix. Note that the columns of this matrix are consisted of corresponding discrete Hermite basis functions. In order to emphasize the visual similarity with considered signals, the first four Hermite basis functions are shown in Fig. 1, with $\sigma=1$.

\section{HERMITE TRANSFORM IN FHSS SIGNAL ANALYSIS}

\section{A. Spread Spectrum in wireless communications}

Commonly applied modulation techniques in communications are the spread spectrum (SS) modulations. Those techniques rely on spreading the frequency spectrum of a data-signal by using a unique code. As a result, the signal with a much higher bandwidth is obtained. This is done to ensure secure data transmission, and to provide robustness to noise, jamming, different types of interferences, etc.

The SS technique has been used by the German military during the World War II [22], to provide secure information exchange.

Two types of SS modulations are commonly used in communications [8], [10], [11], [21]:

- direct sequence spread spectrum (DSSS), where a fast pseudorandom sequence causes phase transitions in the carrier data. This modulation type is used in IEEE $802.11 \mathrm{~b}$ standard for wireless LAN;

- frequency hopping spread spectrum (FHSS), where carrier is caused to shift the frequency in a pseudorandom way [21]. This modulation type is used in Bluetooth standard.

FHSS modulation technique uses a pseudorandom sequence to determine frequencies on which parts of the 
signal appear. Unless the pseudorandom sequence is known, it is hard to assume the frequency at which a carrier wave will appear next.
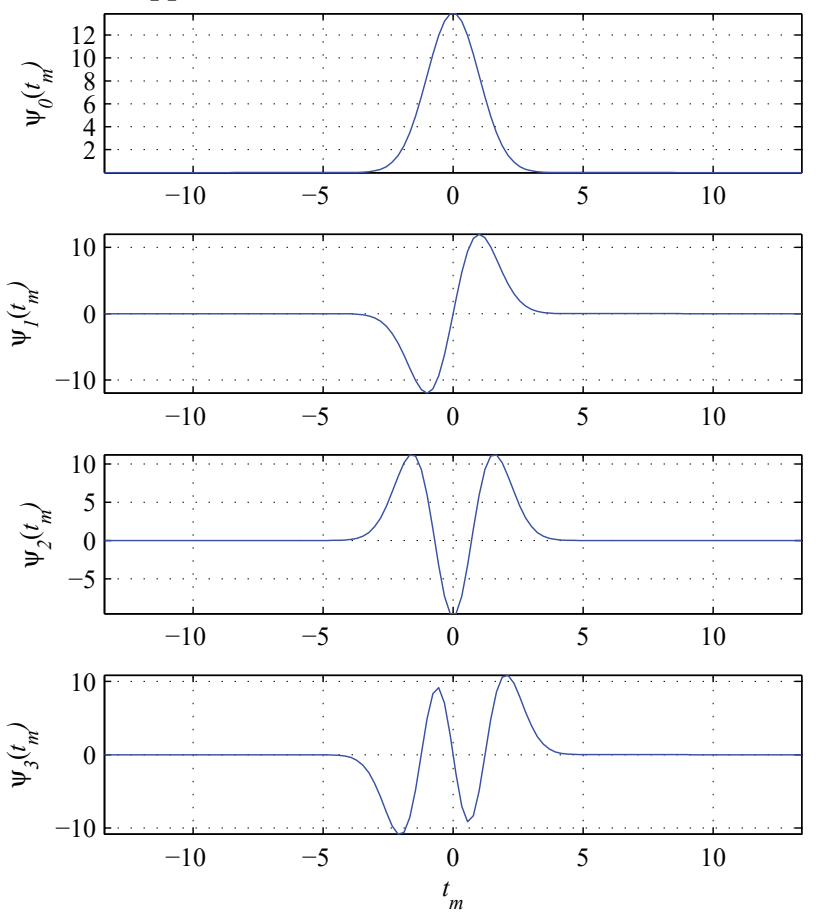

Fig. 1. First four Hermite basis functions.

Since the FHSS modulated signals cannot be represented with a small number of DFT/DCT coefficients, an alternative sparsity domain should be considered. The similarity in the shapes of the FHSS signal components and the Hermite basis function has been exploited. Therefore, the Hermite functions are chosen as a starting basis. The adaptation of Hermite functions to the shapes of the signal components is performed, with a goal of achieving a better sparsity. The adaptation is done in two major steps: Firstly, the widths of Hermite functions are changed in order to fit the widths of signal components. Secondly, signal components are shifted in time to better match the positions of basis functions with respect to the time axis origin. By choosing suitable fitting parameters, the HT of analysed signals can be optimally sparsified, even in the cases when the non-parameterized transform is not inherently sparse.

\section{B. Optimal signal representation in the HT domain}

The discrete signals of length $M$, being represented by the HT, should be sampled at non-uniform points proportional to the roots of the $M$-th order Hermite polynomial, according to (5). Signal samples are usually available on a uniform grid, defined by the sampling theorem. Therefore, signal samples at the points required by the HT should be obtained using an interpolation. It has been shown that a finite $\operatorname{sinc}$ interpolation is suitable for this purpose [12]:

$$
f\left(\beta t_{m}\right) \approx \sum_{n=-K}^{K} f(n \Delta t) \frac{\sin \left(\pi\left(\beta t_{m}-n \Delta t\right) / \Delta t\right)}{\pi\left(\beta t_{m}-n \Delta t\right) / \Delta t},
$$

where $m=1, \ldots, M, n=-K, \ldots, K$ and $\Delta t$ is the sampling period. Instead of stretching and compressing the basis functions, alternatively, we fix $\sigma=1$ in (2) and introduce the signal time-axis scaling factor $\beta$. As the aim is to find the value of the parameter producing the best possible concentration (i.e. sparsity), concentration measure, namely the $\ell_{1}$-norm, is used as the optimization criterion:

$$
\begin{aligned}
\beta_{\text {opt }} & =\min _{\beta}\|\tilde{\mathbf{c}}\|_{1}=\min _{\beta}\left\|\operatorname{HT}\left\{f\left(\beta t_{m}\right)\right\}\right\|_{1}= \\
& =\left\|\operatorname{HT}\left\{\sum_{n=-K}^{K} f(n \Delta t) \frac{\sin \left(\pi\left(\beta t_{m}-n \Delta t\right) / \Delta t\right)}{\pi\left(\beta t_{m}-n \Delta t\right) / \Delta t}\right\}\right\|_{1},
\end{aligned}
$$

where the operator $\operatorname{HT}\{\cdot\}$ is used to denote the Hermite transform of the signal rescaled calculated according to (6) where $\sigma=1$ is assumed in the definition of basis functions (2). Note also that $\|\tilde{\mathbf{c}}\|_{1}$ is used to denote the $\ell_{1}$-norm of the Hermite coefficients $\tilde{\mathbf{c}}$ of the rescaled signal, calculated as:

$$
\|\tilde{\mathbf{c}}\|_{1}=\sum_{p=0}^{M-1}\left|\tilde{c}_{p}\right|
$$

which is used as the HT sparsity measure.

The optimization problem (9) is a 1-D search over the possible values of the scaling factor $\beta$. It was shown that the considered $\ell_{1}$-norm exhibits convexity under conditions considered in detail in [12], where an adaptive iterative algorithm is also proposed to solve (9) without a direct search approach. The adaptive algorithm for finding the parameter $\beta_{\text {opt }}$ follows.

Step 0. The initialization of the parameters is performed. The scaling factor is initialized at:

$$
\beta^{(0)} \leftarrow \frac{M \Delta t}{2(\sqrt{\pi(M-1) / 1.7}+1.8)} .
$$

This starting point ensures that the possible divergence of the algorithm for a too small scaling factor is avoided. This lower bound is introduced in [24] and [25]. A step parameter $\Theta$ is also introduced and initialized as follows:

$$
\Theta \leftarrow 2 / t_{M},
$$

as well as the predefined precision acting as a stopping criterion in the adaptation process:

$$
\varepsilon \leftarrow 10^{-10} \text {. }
$$

Then, until $\Theta>\varepsilon$ is satisfied, the following steps are repeated:

Step 1. Calculate matrices for signal rescaling according to (8), with scaling factors $\lambda+\Theta$ and $\lambda-\Theta$ :

$$
\boldsymbol{\Lambda}_{\beta}^{+} \leftarrow\left[\begin{array}{cccc}
\lambda_{11}^{+} & \lambda_{12}^{+} & \cdots & \lambda_{1 M}^{+} \\
\lambda_{12}^{+} & \lambda_{22}^{+} & \cdots & \lambda_{2 M}^{+} \\
\vdots & \vdots & \ddots & \vdots \\
\lambda_{M 1}^{+} & \lambda_{M 2}^{+} & \cdots & \lambda_{M M}^{+}
\end{array}\right], \boldsymbol{\Lambda}_{\beta}^{-} \leftarrow\left[\begin{array}{cccc}
\lambda_{11}^{-} & \lambda_{12}^{-} & \cdots & \lambda_{1 M}^{-} \\
\lambda_{12}^{-} & \lambda_{22}^{-} & \cdots & \lambda_{2 M}^{-} \\
\vdots & \vdots & \ddots & \vdots \\
\lambda_{M 1}^{-} & \lambda_{M 2}^{-} & \cdots & \lambda_{M M}^{-}
\end{array}\right] \text {, }
$$

having elements

$$
\lambda_{i j}^{ \pm}=\frac{\sin \left[\pi\left((\beta \pm \Theta) t_{i}-(j-K-1) \Delta t\right) / \Delta t\right]}{\pi\left((\beta \pm \Theta) t_{i}-(j-K-1) \Delta t\right) / \Delta t},
$$

with $i, j \in\{1,2, \ldots, M\}$.

Step 2. Calculate the measures of sparsity for signal resampled with $\beta+\Theta$ and $\beta-\Theta$ employing the matrices $\boldsymbol{\Lambda}_{\lambda}^{+}$and $\boldsymbol{\Lambda}_{\lambda}^{-}$from step 1: 


$$
\mathcal{M}^{+} \leftarrow\left\|\mathbf{c}^{+}\right\|_{1}=\sum_{p=0}^{M-1}\left|\mathbf{H} \mathbf{\Lambda}_{\beta}^{+} \mathbf{f}\right|, \mathcal{M}^{-} \leftarrow\left\|\mathbf{c}^{-}\right\|_{1}=\sum_{p=0}^{M-1}\left|\mathbf{H} \mathbf{\Lambda}_{\beta}^{-} \mathbf{f}\right|
$$
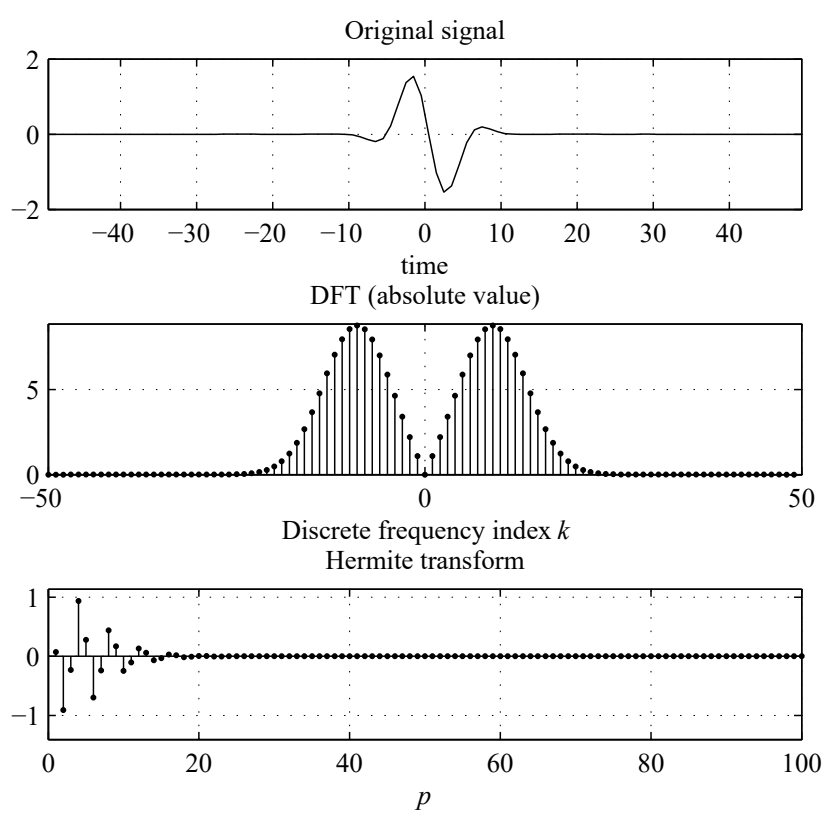

Optimized Hermite transform

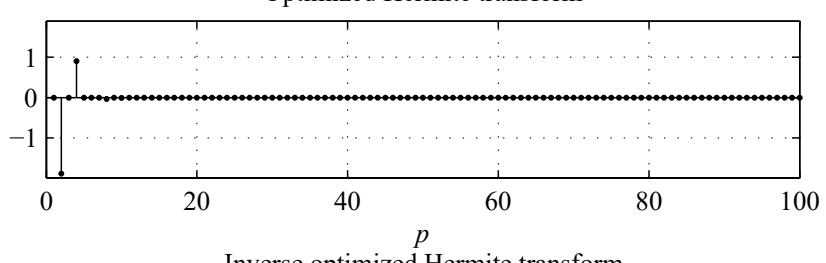

Inverse optimized Hermite transform

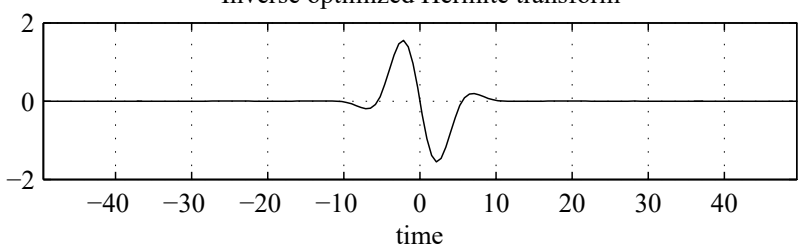

Fig. 2. Hermite transform optimization for a windowed sinusoid: first row - original signal in time domain, second row - DFT of the considered signal, third row - HT of the considered signal, fourth row - HT with optimized scaling factor, last row - signal reconstructed using the optimized HT.

Step 3. Approximate the scaling factor gradient using the difference of the measures from step 2 :

$$
\nabla^{(k)} \leftarrow \frac{\mathcal{M}^{+}-\mathcal{M}^{-}}{M}
$$

Step 4. Update the scaling factor in a steepest descent manner:

$$
\beta^{(k+1)} \leftarrow \beta^{(k)}-\mu \nabla^{(k)}
$$

Here $k$ is the iteration index, and $\mu=0.05$ is used for FHSS signals. When the stopping criterion is met, the optimal scaling factor $\beta$ is returned. A high precision of the gradient approximation in (16) is assured by an iterative reduction of the step $\Theta$. It occurs when further updates using scaling factors $\beta+\Theta$ and $\beta-\Theta$ do not reduce the concentration measures (15).

Namely, in that case, the gradient (16) leads to the oscillations around the optimal point, which cannot be reached as the step $\Theta$ is too large. In this case, during the two consecutive iterations $(k-1)$ and $(k)$ the gradient changes its sign (16). This can be detected using the sign of the gradients product from two consecutive iterations:
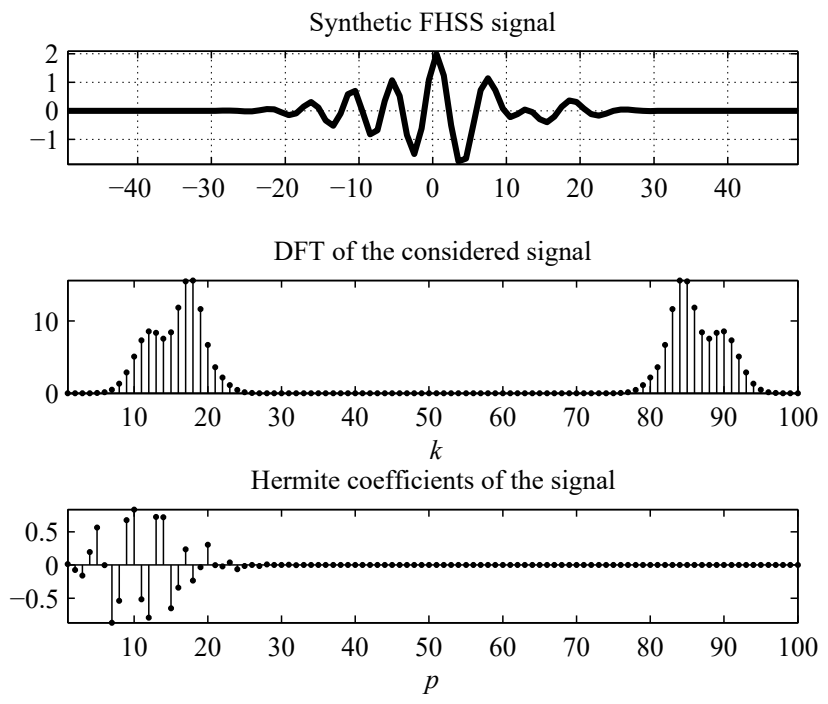

Fig. 3. The considered three-component FHSS signal: first row time domain, second row - DFT, third row - HT domain.
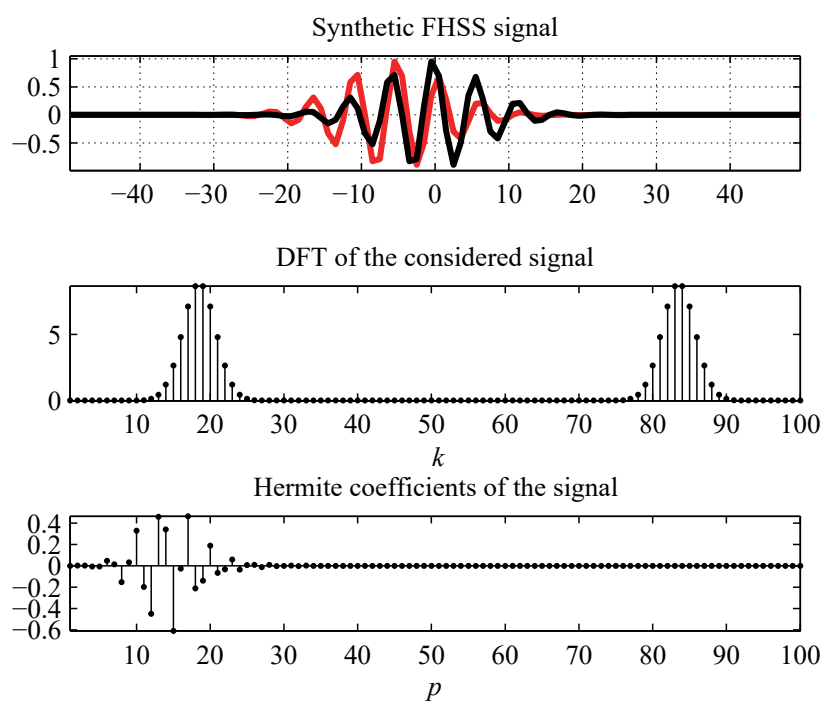

$p$
Coefficients of the sparsified Hermite transform

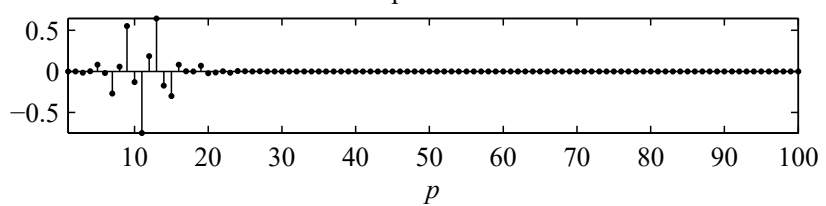

Inverse optimized Hermite transform

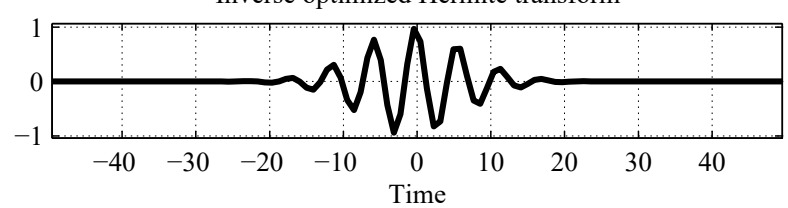

Fig. 4. The first component in the FHSS signal shown in Fig.1: original (first row, full line) and optimally shifted component (first row, solid red line), DFT, HT and optimized HT (second fourth rows), component reconstructed from the optimized HT domain (fifth row).

$$
\alpha \leftarrow \operatorname{sign}\left(\nabla^{(k)} \nabla^{(k-1)}\right)
$$

When the gradient sign changes during the two consecutive iterations, then $\alpha<0$. When this condition is satisfied, the step should be reduced:

$$
\Delta \leftarrow \Delta / 2
$$


The gradient sign check and step reduction when $\alpha<0$ should be done in each iteration $k$.

In a similar way, after the extraction of FHSS localized components $f_{i}(n \Delta t)$, instead of $f_{i}(n \Delta t)$ shifted signals $f_{i}((n \pm \phi) \Delta t)$ can be used in (8), with $\phi \in\left[-\phi_{\max }, \phi_{\max }\right]$. This is a small integer shift left or right from the origin. For every possible $\phi$ optimization (9) is done, and a measure vector $\mathbf{M}$ is formed. After that, the shift producing the minimal concentration measure is selected, according to:

$$
\phi=\underset{\phi}{\arg \min } \mathbf{M} \text {. }
$$

\section{NUMERICAL RESULTS}

\section{A. Illustration of the scaling factor optimization}

In this example, we illustrate the influence of the scaling factor on the HT coefficients concentration, for the case of a uniformly sampled signal. In the optimization, the signal is resampled at points proportional to the roots of the Hermite polynomial.

Consider the windowed form of the sinusoidal signals (Gaussian window is applied):

$$
f(n)=-2 \sin (18 \pi n / M) e^{-\frac{n}{2 \sigma_{0}^{2}}}
$$

with $\sigma_{0}=3.5,-M / 2 \leq n \leq M / 2-1$. The signal sparsity in the HT domain is significantly improved when compared with the non-optimized HT (Fig. 2, the third and fourth rows). The considered optimized Hermite transform (Fig. 2, the fourth row) is the most optimal transform for windowed sinusoids, unlike the Fourier transform which is spread over half of the frequency band Fig. 2, the second row.

\section{B. FHSS signal sparsification}

Multi-component (hops) FHSS is considered as the signal model. This synthetic model can be described by:

$$
f(n)=\sum_{i=1}^{K} A_{i} e^{-\left(\frac{n-\tau_{i}}{\gamma_{i}}\right)^{2}} \cos \left(\omega_{i} n\right),
$$

with $K=3, \omega_{i} \in\{1.4 \pi / 4,0.91 \pi / 4,1.25 \pi / 4\}, \quad \gamma_{i}=10$, $A_{i}=1$ and $\tau_{i} \in\{M / 16,-M / 15,-M / 16\}$ for $i=1,2,3$, respectively. The signal length is $M=100$ samples. The hops have the same duration, and they differ in frequency. The component shift from the origin is denoted by $\tau_{i}$.

The time domain as well as the DFT and the HT of the signal are shown in Fig. 3. It can be noticed that the number of nonzero HT coefficients (or coefficients with significant values) is smaller than in the DFT case.

However, individual components are better concentrated, and therefore, the signal can be firstly decomposed [10], and then the separated components are further considered.

The shape resemblance of the separated components with the Hermite basis functions is an indication of a potential sparsity representation and possible application of the approach [12]. This is confirmed by the results shown in Figs 4 and 5, illustrating the fact that the HT exhibits a better concentration when compared to DFT. It is further improved incorporating the presented optimizations of the scaling factor and time shift.
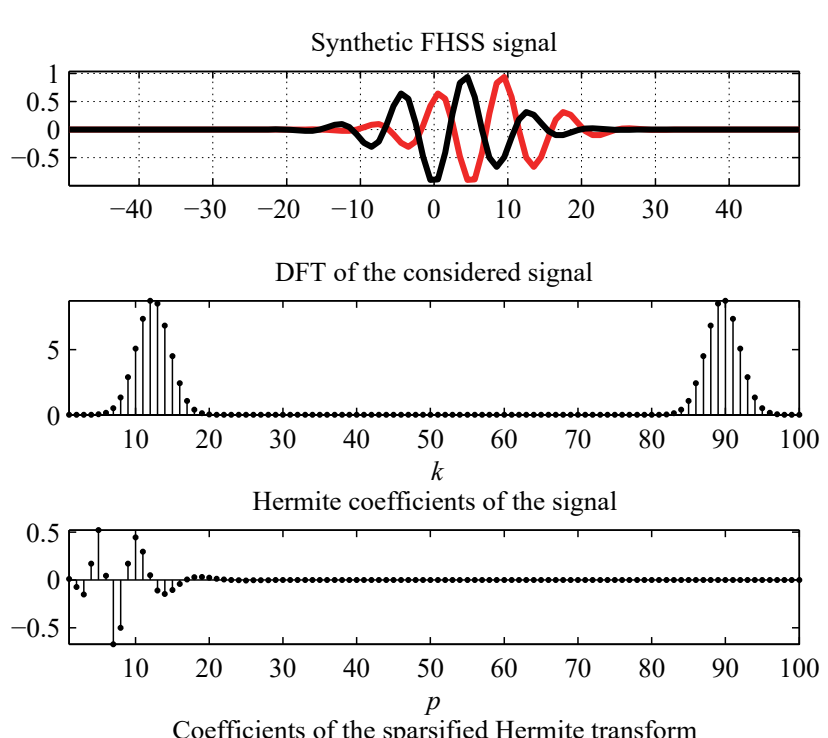

Coefficients of the sparsified Hermite transform

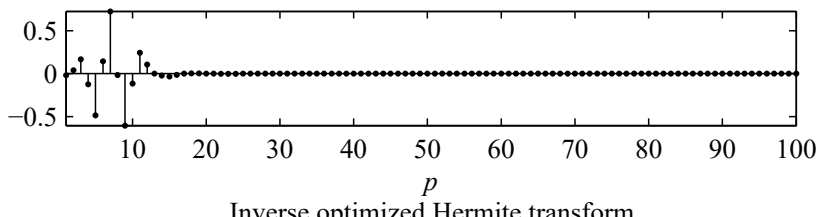

Inverse optimized Hermite transform
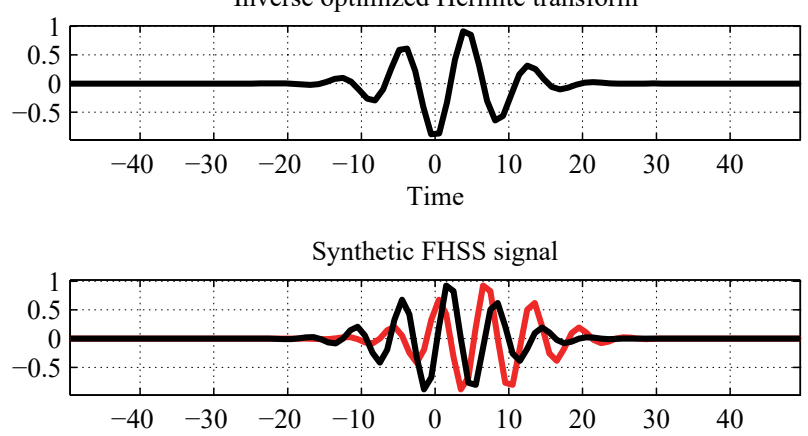

DFT of the considered signal
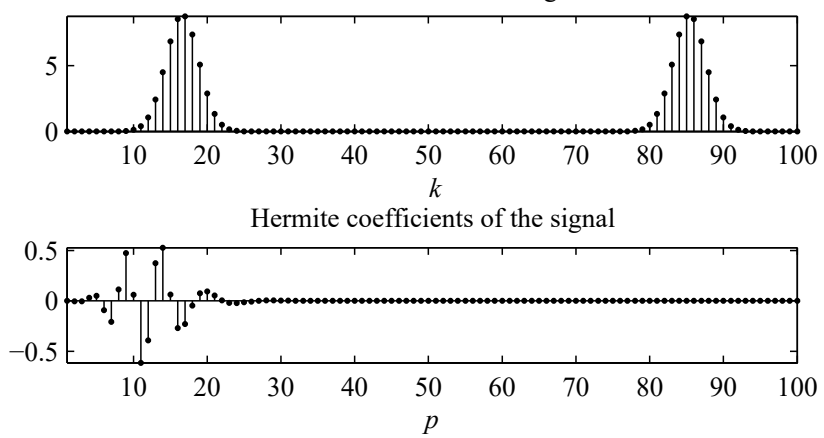

Coefficients of the sparsified Hermite transform

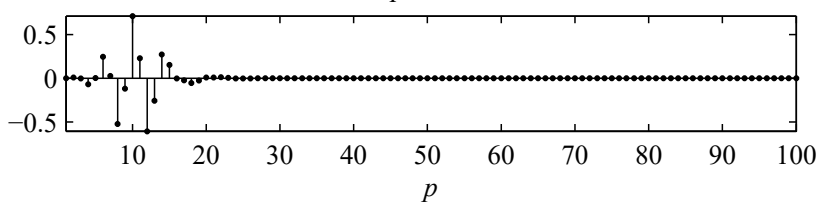

Inverse optimized Hermite transform

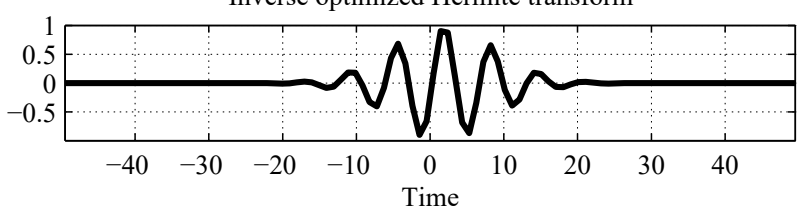

Fig. 5. The second and the third component of the FHSS signal defined in (22): original ( $1^{\text {st }}$ and $6^{\text {th }}$ rows, full line) and optimally shifted components ( $1^{\text {st }}$ and $6^{\text {th }}$ rows, solid red line $)$, DFT ( $2^{\text {nd }}$ and $7^{\text {th }}$ row $)$, HT ( $3^{\text {rd }}$ and $8^{\text {th }}$ row) and optimized HT ( $4^{\text {th }}$ and $9^{\text {th }}$ row), component reconstructed from the optimized HT domain $\left(5^{\text {th }}\right.$ and $10^{\text {th }}$ rows $)$. 

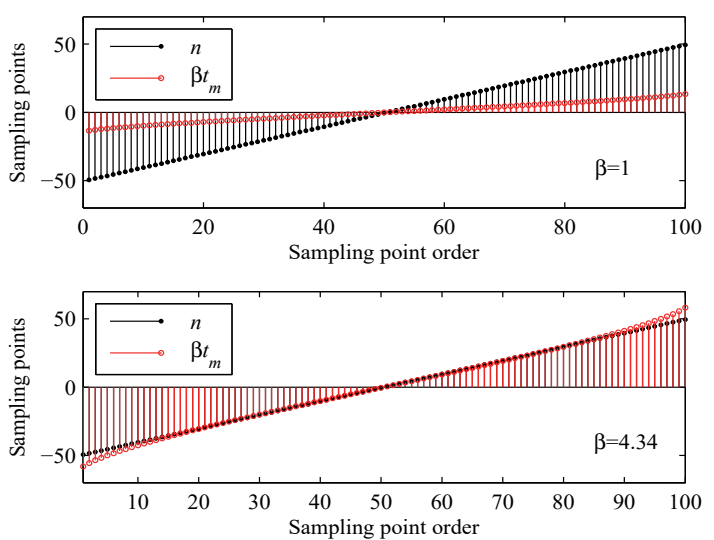

Fig. 6. Uniform discrete time grid and the time grid formed based on zeros of 100-th order Hermite polynomial for a signal with length $M=100$. Two scaling factors are used: unity and the optimal one.

The influence of the scaling factor choice on the positioning of Hermite polynomial roots and the nonuniform time grid formation is illustrated in Fig. 6, for signal (22) shown in Fig. 5. Red dots represent non-uniform sampling grids obtained based on roots of the $M$-th order Hermite polynomial $(M=100)$, whereas black dots represent uniform sampling grids obtained based on the sampling theorem. In Fig. 6, the first subplot, the grids are compared when $\beta=1$. Non-uniform (red) and uniform (black) grids significantly differ, consequently leading to a non-optimal signal representation. In Fig. 6, the second subplot, scaling factor $\beta=4.34$, being the output of the presented algorithm, is used to form the non-uniform grid (red), whose points now highly match the uniform grid (black). We can conclude that the improvement of the sparsity is related to the placement of the non-uniform grid points in the vicinity of uniform sampling grid points.

\section{CONCLUSION}

The Hermite transform domain is considered as a domain of sparsity for FHSS communication signals. Optimization of scaling factor and time shift are incorporated for the FHSS sparsification in this particular domain. Multicomponent signals are decomposed into single components, and each component separately is further processed. The optimization of the transform domain is done by minimizing the $\ell_{1}$-norm based concentration measure. The results indicate further applicability in compressed sensing scenarios and sparse signal reconstruction algorithms.

\section{REFERENCES}

[1] F. Marvasti, A. Amini, F. Haddadi, M. Soltanolkotabi, B. Hossein Khalaj, A. Aldroubi, S. Sanei, J. Chambers, "A unified approach to sparse signal processing", EURASIP Journal on Advances in Signal Processing 20122012:44.

[2] S. Stanković, I. Orović, LJ. Stanković, "Compressive Sensing approach in Hermite transform domain," Math Problems in Engineering, Volume 2015 (2015), Article ID 286590, 9 pages.

[3] R. E. Carrillo, K. E. Barner, T. C. Aysal, "Robust sampling and reconstruction methods for sparse signals in the presence of impulsive noise," IEEE Journal of Selected Topics in Signal Processing, vol. 4, no.2, pp. 392-408, 2010.

[4] I. Orovic, S. Stankovic, "Improved Higher Order Robust Distributions based on Compressive Sensing Reconstruction," IET Signal Processing, Vol. 8, No. 7, pp. 738 - 748, May 2014.

[5] S. Stankovic, I. Orovic, E. Sejdic, Multimedia Signals and Systems, Springer-Verlag, New York, 2012.

[6] M. Elad, Sparse and Redundant Representations: From Theory to Applications in Signal and Image Processing, Springer 2010.

[7] I. Orovic, A. Draganic, S. Stankovic, "Sparse Time-Frequency Representation for Signals with Fast Varying Instantaneous Frequency," IET Radar, Sonar \& Navigation, vol. 9, issue 9, pp. $1260-1267,2015$.

[8] M. Gandetto, M. Guainazzo, C. S. Regazzoni, "Use of TimeFrequency Analysis and Neural Networks for Mode Identification in a Wireless Software-Defined Radio Approach", EURASIP Journal on Applied Sig. Process., Vol. 2004, pp. 1778-1790, 2004.

[9] S. Stankovic, I. Orovic, B. Mobasseri, M. Chabert, "A Robust Procedure for Image Watermarking based on the Hermite Projection Method," Automatika - Journal for Control, Measurement, El., Comp. and Commun., vol 53, no 4, 2012.

[10] A. Draganic, I. Orovic, S. Stankovic, "FHSS Signal Characterization Based On The Crossterms Free Time-Frequency Distributions," 2nd Mediterranean Conference on Embedded Computing MECO-2013, pp. 152-155, June 2013, Budva, 2013.

[11] O. Berder, C. Bouder, G. Burel, "Identification of Frequency Hopping Communications", Problems in Modern App. Mathem., published by WSES, 2000, pp. 259-264, ISBN 960 8052-15-7.

[12] M. Brajović, I. Orović, M. Daković, S. Stanković, "On the Parameterization of Hermite Transform with Application to the Compression of QRS Complexes," Signal Processing, vol. 131, pp. 113-119, 2017.

[13] A. Sandryhaila, S. Saba, M. Püschel, J. Kovačević, "Efficient Compression of QRS Complexes Using Hermite Expansion," IEEE Trans. on Sig. Processing, vol. 60, no. 2, pp. 947-955, Feb. 2012.

[14] M. Brajović, I. Orović, M. Daković, S. Stanković, "Gradient-based signal reconstruction algorithm in Hermite transform domain," Electronics Letters, vol. 52, no. 1, pp. 41-43, 182016.

[15] M. Brajović, I. Orović, S. Stanković, "The Optimization of the Hermite transform: Application Perspectives and 2D Generalization," 24th Telecommunications Forum TELFOR 2016, November 2016, Belgrade, Serbia.

[16] A. I. Rasiah, R. Togneri, Y. Attikiouzel, "Modelling 1-D signals using Hermite basis functions," IEE Proceedings - Vision, Image and Signal Processing, vol. 144, no. 6, pp. 345-354, Dec. 1997.

[17] J.-B. Martens, "The Hermite transform-theory," IEEE Trans. on Ac., Speech and Sig. Proc., vol.38, no.9, pp.1595-1606, 1990.

[18] M. Brajovic, A. Draganic, I. Orovic, S. Stankovic, "FHSS signal sparsification in the Hermite transform domain," 24th Telecommunications Forum TELFOR 2016, Nov. 2016, Belgrade.

[19] S. Stanković, I. Orovic, A. Krylov, "The Two-Dimensional Hermite S-method for High Resolution Inverse Synthetic Aperture Radar Imaging Applications," IET Sig. Process. vol. 4, no. 4, pp: 352-362, 2010.

[20] A. Krylov, D. Korchagin, "Fast Hermite projection method," 3rd Int. Conference on Image Analysis and Recognition (ICIAR '06), vol. 1, pp. 329-338, Povoa de Varzim, Portugal, Sept. 2006.

[21] R. Pickholtz, D. Schilling, L. Milstein, "Theory of Spread-Spectrum Communications - A Tutorial," in IEEE Transactions on Communications, vol. 30, no. 5, pp. 855-884, May 1982.

[22] G. Klemens, The Cellphone: The History and Technology of the Gadget That Changed the World, McFarland, Sept. 2010

[23] I. Orović, V. Papić, C. Ioana, X. Li, S. Stanković, "Compressive Sensing in Signal Processing: Algorithms and Transform Domain Formulations," Mathem. Problems in Eng., Review paper, 2016.

[24] R. Ma, L. Shi, Z. Huang, Y. Zhou, EMP Signal Reconstruction Using Associated-Hermite Orthogonal Functions, IEEE Trans. on Electromagnetic Compatibility 56 (5) (2014) 1242-1245

[25] M. Yuan, A. De, T. K. Sarkar, J. Koh, B. H. Jung, "Conditions for generation of stable and accurate hybrid TD-FD MoM solutions," IEEE Trans. on Microwave Theory and Techniques 54 (6) (2006) 2552-2563. 